\title{
Left Sided Boshdalek Hernia in Adult: A Case Report
}

\section{Antonio Gligorievski*}

*Correspondence: Antonio Gligorievski

Address: University Clinic of Surgery "St. Naum Ohridski" Skopje, Macedonia

e-mail $\bowtie$ : atglmed@gmail.com

Received: 23 February 2020; Accepted: 05 March 2020

Copyright: (C) 2020 Gligorievski A. This is an open-access article distributed under the terms of the Creative Commons Attribution License, which permits unrestricted use, distribution, and reproduction in any medium, provided that the original work is properly cited.

\begin{abstract}
Introduction: Congenital left diaphragmatic hernia of Bochdalek rarely occurs in adults. There are fewer than 100 cases of left-sided Bochdalek hernia reported in adults in the literature. Most of them are asymptomatic. Bochdalek hernias typically arise on the left side, contain fat or omentum, stomach, part of colon transverse, predominantly and small intestinal loops.

Aim: The aim of this paper is to present a rare case of Bochdalek hernia in adults and to point out the important role of Computed tomography (CT) in reaching the exact diagnosis of this abnormality.

Case report: We presented a rare case of a 46-year old female with left-sided Bochdalek diaphragmatic hernia, came to the Clinic of Surgery complaining of abdominal pain in the lower abdomen. The chest radiograph showed features suggestive of left-sided diaphragmatic hernia, which was confirmed using Multi-Dimensional Computed Tomography (MDCT).

Conclusion: Bochdalek hernia in adults occurs most frequently when the patients are undergoing CT for reasons that appear to be unrelated to the hernia.
\end{abstract}

Keywords: Adult, Boshdalek, Computed Tomography, Congenital, Diaphragmatic, Hernia

\section{Introduction}

Congenital diaphragmatic hernias are categorized as poster lateral (Bochdalek), anteriorretrosternal (Morgagni), and Hiatal hernias. The first congenital diaphragmatic hernia was described clinically by Vincent Alexander Bochdalek in 1848 (Salacin et al., 1994). Bochdalek hernia involves the posterior defect in diaphragm caused by the defective fusion of the pleuroperitoneal membrane (Mullins et al., 2001). Bochdalek hernia is the most common type of diaphragmatic hernia, accounting for $95 \%$ of cases. In adults, most Bochdalek hernias are asymptomatic, and therefore their detection is usually incidental (Ameet et al., 2009). Diagnosis of a Bochdalek hernia in an adult is rare and is typically observed on the left side of the diaphragm, $85 \%$ of cases (Ameet et al., 2009). An adult Bochdalek hernia is usually precipitated by a state of increased intra-abdominal pressure, such as that induced by pregnancy, or blunt injury, physical effort, coughing episodes, sneezing, and even eating too much food. Reports of Bochdalek hernias in adults remain limited to single patients or small series. Approximately 150 cases of an adult left-sided Bochdalek hernia can be found in the world literature. The widespread use of multi-detector row computed tomography (MDCT) for the evaluation of thoracic and abdominal 
disease has resulted in a significant increase in the incidental discovery of occult lesions. The current treatment of choice of a Bochdalek hernia is surgical repair even in asymptomatic cases because of the risk of visceral herniation and strangulation.

\section{Presentation of Case}

The aim of this report is to point out the significance of clinical suspicion and the important role of imaging techniques in reaching the exact diagnosis of congenital diaphragmatic hernia. We present a rare case of 46 years old female with an incidentally detected congenital diaphragmatic hernia on the left side and to emphasize the significant role of imaging for the diagnosis of this condition. She complained about epigastric and abdominal pain for one week and a moderate form of dyspnea. There was no history of any previous abdominal or thoracic trauma. She had no other underlying disease. Her physical examination revealed pain in the epigastric area, but did not reveal any significant abnormality. She was sent to the Radiology Department for further evaluation. Posteroanterior (PA) and latero-lateral (LL) chest X-ray revealed that the left diaphragm was unexceptionally high (Fig. 1). MDCT scan of the chest and abdomen was done. MDCT scan of the chest and abdomen revealed the presence of a left-sided postero-lateral diaphragmatic defect with herniation of the stomach and part of the left transverse colon including mesenteric fat into the left hemithorax (Fig. 2-4). The herniated bowel passes through the defect, filling the chest cavity and causing the compression of the lower lobe of the lung, and a shift of mediastinal structures to the right side. MDCT scan confirmed the diagnosis of a left-sided Bochdalek hernia (Figure 2, $3,4)$. The patient was counseled about the possible complications of diaphragmatic hernia. After repeated counseling explaining the possible complications that might occur, if left untreated, the patient still refuses surgery.
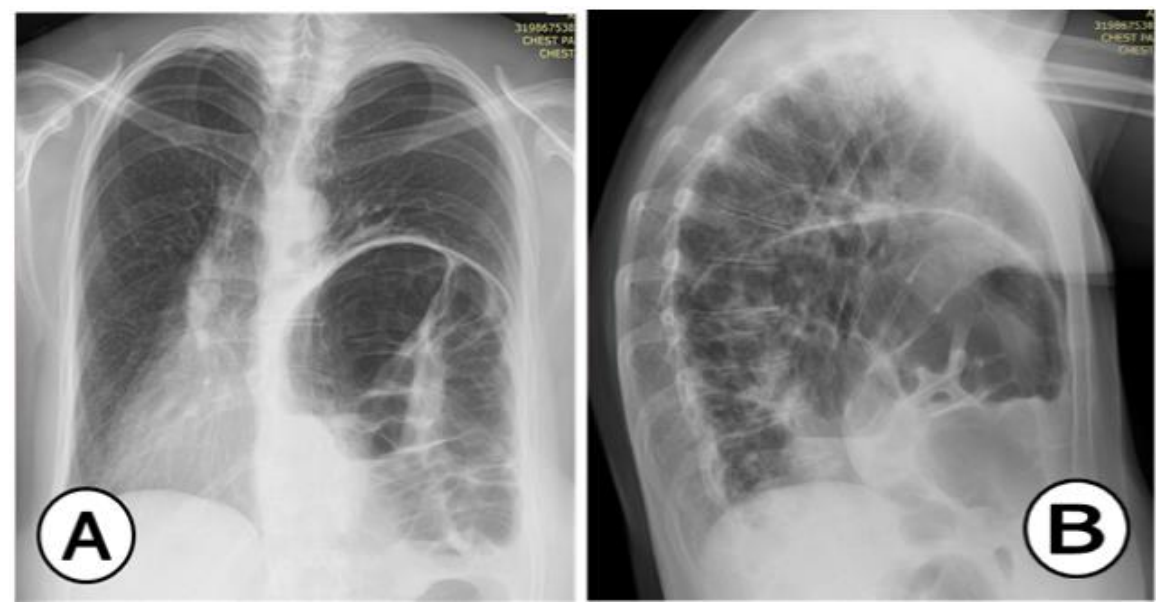

Figure 1: Radiographic images of the adult left-sided Bochdalek hernia. A) PA chest X-ray and B) LL chest X-ray: Showed an elevated left hemidiaphragm, and there was also suspicious of herniation of stomach and part of the left transverse colon including small intestine in left hemithorax. Noted the right-sided shift of the heart and mediastinum. 

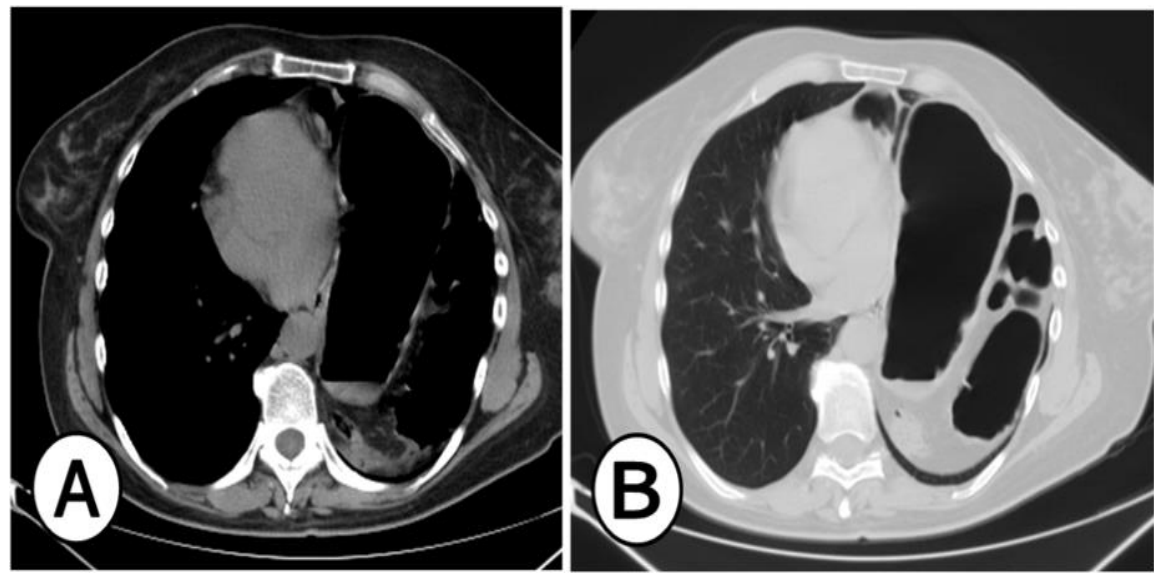

Figure 2: Noncontrast CT scans of the thorax and abdomen. A) Mediastinal window; B) Lung window: the level of basal ganglia and lateral ventricles (A) and (B): Axial CT images clearly show poster lateral left hemidiaphragm defect with herniation of the left transverse colon, stomach, small intestine, and intraperitoneal fat into the left hemithorax.

This present case highlights the importance of early CT scanning in reaching an early preoperative diagnosis, particularly since a delay in diagnosis can result in significant morbidity and mortality.
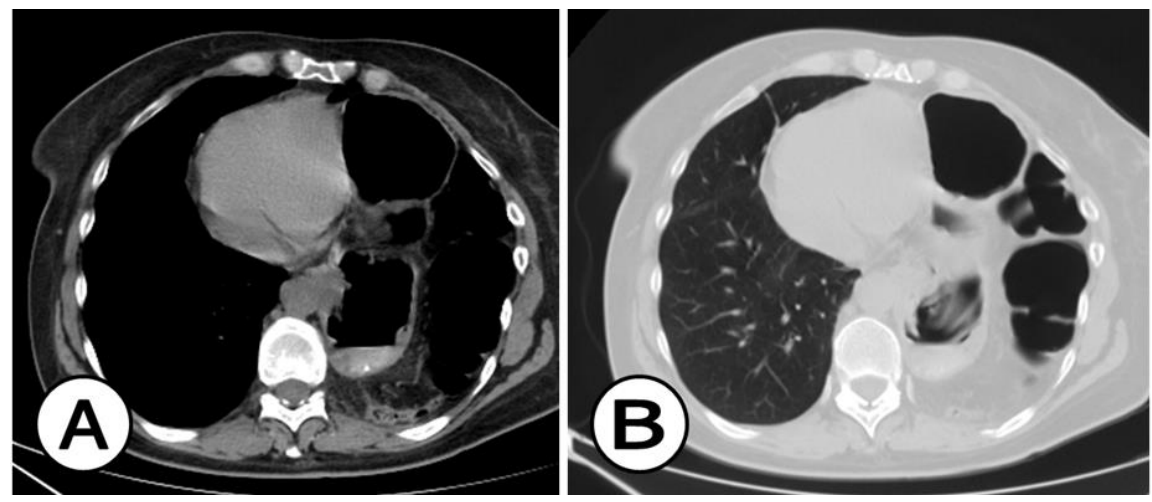

Figure 3: Noncontrast CT scans of the thorax and abdomen. A) Mediastinal window; B) Lung window: the level of basal ganglia and lateral ventricles (A) and (B): Axial CT images clearly show herniation of the stomach, left transverse colon, small intestine, and intraperitoneal fat into the left hemithorax in 46 years old woman.
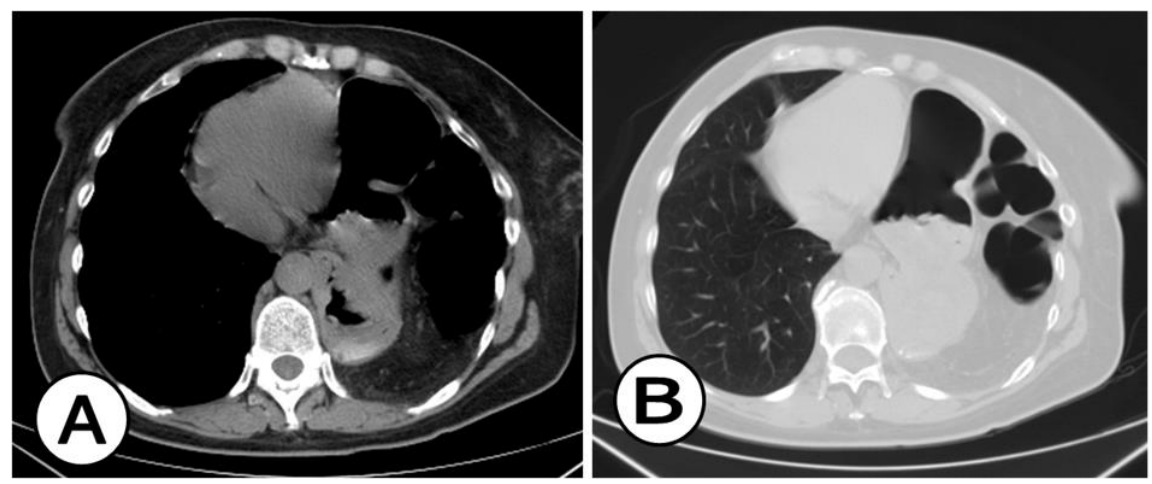

Figure 4. Noncontrast CT scans of the thorax and abdomen. A) Mediastinal window; B) Lung window: the level of basal ganglia and lateral ventricles (A) and (B): Axial CT images clearly show herniation of the stomach, left transverse colon, small intestine, and intraperitoneal fat into the left hemithorax in 46 years old woman. 


\section{Discussion}

Congenital diaphragmatic hernias, clinically represented in adult life are extremely rare, and they can occur through the frontal parasternal opening (Morgagni hernia 1\% to 6\%) or through the poster lateral opening (Bochdalek's hernia 80\% to 90\%) (Ameet et al., 2009). Poster lateral, Bochdalek hernia is the most common type of congenital diaphragmatic hernias that occurs in $95 \%$ of cases, resulting from inadequate closure of the poster lateral pleuroperitoneal membranes (Salacin et al., 1994). The overall prevalence of Bochdalek hernias in adults is 6\% to 10\% (Ilkins et al., 1994; Gale, 1995). The literature reports that defects occur more frequently on the left side (80-90\%) than on the right side of the diaphragm (Gale, 1995; Nitecki and Mar-Maor, 1992), and the hernia contents included omentum and fat but also the splenic flexure of the colon, stomach, small bowel loops and spleen (Wilbur et al., 1994; Mehdi et al., 1995). In adults, most Bochdalek's hernias are asymptomatic, and thus the finding of the condition is incidental (Fine et al., 1987; Langer, 1998). Some patients have no symptoms and the disorder is unexpectedly detected on a chest X-ray or CT scan of the thorax (Shin et al., 1987). Unclear and nonspecific clinical presentation results in late diagnosis. The diagnosis of Bochdalek hernia in adulthood is problematic because of the rarity of this disease and the variety of the presenting symptoms. Symptomatic cases of Bochdalek's hernia in adults are fairly rare. Adult Bochdalek hernia commonly presents with gastrointestinal symptoms such as abdominal pain, abdominal distension, dysphagia and vomiting due to obstruction of the prolapsed gastrointestinal tract (Losanoff and Sauter, 2004). Although the adult lungs are fully developed, the symptom of impaired respiration because of adult Bochdalek hernia can still occur, and usually include dyspnea and chest pain (Wilbur et al., 1994; Hines and Romero, 1983). They are diagnosed accidentally in adults, as an incidental medical finding or when the symptoms appear. Our patient presented with abdominal pain. Symptoms may be intermittent or acute depending on the extent of herniation of abdominal viscera into the thorax. Acute presentation is usually due to incarceration, obstruction, or strangulation of the hernia (Hung et al., 2008; Kocakusak et al., 2005). Diagnosis is ascertained by a combination of chest X-rays, computed tomography (CT), as well as upper gastrointestinal and bowel double-contrast study. Imaging plays an important role in diagnosing and assessing the contents of the hernia and at the same time evaluating the presence of any associated abnormality (Irish et al., 1996). The incidence of Bochdalek's hernia in routine CT scans has been reported to vary from $0.17 \%$ to $6 \%$ (Temizoz et al., 2010). The size of the hernia seen on cross-sectional imaging does not necessarily correspond to the size of the diaphragmatic defect, which may be substantially larger (Temizoz et al., 2010). Although adult Bochdalek hernia has the potential to be a very morbid condition, the diagnosis can be made with a careful radiographic examination.

These hernias are evidenced by abnormal findings above the dome of the diaphragm, such as gas- 
filled loops of bowel or soft tissue mass. A Bochdalek hernia can also mimic as a tension pneumothorax on the chest X-ray, which can complicate the treatment (Dalton et al., 2004). Chest CT more accurately visualizes focal defects in the diaphragm and can definitively diagnose herniation in comparison to plain film chest radiography. Typical findings are fat or soft tissue contour on the upper surface of the diaphragm. Another characteristic of a Bochdalek hernia is its poster lateral location. These findings were also present in our patient. The gold standard for diagnosis of a Bochdalek hernia is multidetector CT (MDCT) (Temizoz et al., 2010). Chest radiographs have a sensitivity of greater than $70 \%$ and usually demonstrate air-fluid levels. A study by Killeen et al found CT to have sensitivities of 78\% for left-sided hernias and 50\% for right-sided hernias (Killeen et al., 1999). The diagnosis in our patient was ascertained by a combination of chest X-ray and CT.

The treatment of Bochdalek hernia is surgical, usually without recidivism and patients are usually without symptoms after the intervention. Bochdalek hernia repair can be performed via laparotomy and or thoracotomy (Esmer et al. 2008). Several authors suggested an abdominal approach for left-sided defects and a thoracic approach for right-sided hernias (Yamaguchi et al., 2002). The diaphragmatic defect itself is typically repaired by simple suture closure, implantation of a prosthetic mesh repair, or use of a muscle flap (Thomas and Kapur, 1991). An absorbable suture has the advantage of minimizing the infection risk. On the other hand, no absorbable suture has the advantage of preventing recurrence.

\section{Conclusions}

A diagnosis of an adult diaphragmatic hernia should always be kept in mind in patients with overlapping abdominal and respiratory symptoms with or without a history of trauma. Adult Bochdalek hernias more commonly present with gastrointestinal symptoms than with pulmonary symptoms. Adult left-sided Bochdalek hernias are extremely rare. PA and LL chest radiography is a good screening tool but CT scanning has higher sensitivity for these lesions. However, this diagnosis should be suspected in patients who have been exposed to factors that have increased their intra-abdominal pressure. Signs and symptoms can be non-specific and the present case highlights the importance of acquiring a CT scan should be done in patients with doubtful complicated presentation and before planning any surgical intervention. This is crucial in the management of these patients, as a delay in diagnosis can increase the risk of mortality.

Conflicts of Interest: Dr. Antonio Gligorievski declares that there is no conflict of interest.

Funding: In this case report, there are no sponsors.

Ethical Approval: Ethical approval was not necessary for this case report. 


\section{References}

Ameet K, Vikas M, Ramakrishnan TS, Samaresh S. Caecal perforation with faecal peritonitis - unusual presentation of Bochdalek hernia in an adult: a case report and review of literature. World J Emerg Surg 2009; 4:16.

Dalton AM, Hodgson RS, Crossley C. Bochdalek hernia masquerading as a tension pneumothorax. Emerg Med J 2004, 21: 393-394.

Esmer D, Alvarez-Tostado J, Alfaro A, Carmona R, Salas M. Thoracoscopic and laparoscopic repair of complicated Bochdalek hernia in adult. Hernia 2008; 12: 307-309.

Fine R, Borrero E, Stone A. Bochdalek hernia in adulthood. N Y State J Med 1987; 87: 516-518.

Gale ME. Bochdalek hernia: prevalence and CT characteristics. Radiology. 1985; 156: 449-452.

Hines GL and Romero C. Congenital diaphragmatic hernia in the adult. Int Surg 1983; 68: 349-351.

Hung YH, Chien YH, Yan SL, Chen MF. Adult Bochdalek hernia with bowel incarceration. J Chin Med Assoc 2008; 71: 528531.

Ilkins AC, Govodes GF, Hibbeln JF. Imaging findings in adult Bochdalek hernias. Clin Imaging 1994; $18: 224$ - 229.

Irish MS, Holm BA, Glick PL. Congenital diaphragmatic hernia: historical review. Clin Perinatol 1996; 23: 625-653.

Killeen KL, Mirvis SE, Shanmuganathan K. Helical CT of diaphragmatic rupture caused by blunt trauma. Am J Radiol 1999; 173: 1611-1616.

Kocakusak A, Arikan S, Senturk O, Ycel AF. Bochdalek's hernia in an adult with colon necrosis. Hernia 2005; 9: 284-287.

Langer JC. Congenital diaphragmatic hernia. Chest Surg Clin N Am 1998; 8: 295-314.

Losanoff JE and Sauter ER. Congenital posterolateral diaphragmatic hernia in an adult. Hernia 2004; 8: 83-85.

Mehdi A, Closset J, el Nakadi I, Houben JJ, Veys I, Lambilliotte JP. Hernia of the diaphragm: a clinical case and review of the literature. Acta Chir Belg 1995; 95: 281-283.

Mullins ME, Stein J, Saini SS, Mueller PR. Prevalence of incidental Bochdalek's hernia in a large adult population. AJR Am J Roentgenol 2001; 177: 363-366.

Nitecki S and Mar-Maor JA. Late presentation of Bochdalek hernia:our experience and review of the literature. Isr J Med Sci 1992; 28: 711-714.

Salacin S, Alper B, Cekin N, Gulmen MK. Bochdalek hernia in adulthood: a review and an autopsy case report. J Forensic Sci 1994; 39: 1112-1116.

Shin MS, Mullign SA, Baxley WA, Ho KJ. Bochdalek hernia of diaphragm in the adult. Diagno-sis by computer tomography. Chest 1987, 92: 1098-1101.

Temizoz 0, Gençhellaç H, Yekeler E. Prevalence and MDCT characteristics of asymptomatic Bochdalek hernia in adult population. Diagn Interv Radiol 2010; 16: 52-55.

Thomas S and Kapur B. Adult Bochdalek hernia: clinical features, management and results of treatment. Jpn J Surg 1991; 21: 114-119. 
Wilbur AC, Gorodetsky A, Hibbeln JF. Imaging findings of adult Bochdalek hernias. Clin Imaging 1994; 18: $224-229$.

Yamaguchi M, Kuwano H, Hashizume M, Sugio K, Sugimachi K, Hyoudou Y. Thoracoscopic treatment of Bochdalek hernia in the adult: report of a case. Ann Thorac Cardiovasc Surg 2002; 8: 106-108. 